\title{
A review on the most important management of keratocystic odontogenic tumor
}

\author{
Přehled nejdůležitějších metod léčby keratocystického \\ odontogenního tumoru
}

\author{
Azadi M. ${ }^{1}$, Bashar S. ${ }^{2}$, Hajiani N. ${ }^{1}$, Amiri H. ${ }^{1}$, Azadi S. ${ }^{3}$, Ansari Z. ${ }^{4}$ \\ 'Resident of Oral and Maxillofacial Surgery, Faculty of Dentistry, Tehran University of Medical sciences, Tehran, Iran \\ 2 Department of Oral and Maxillofacial Surgery, Faculty of Dentistry, Golestan University of Medical Sciences, Gorgan, Iran \\ ${ }^{3}$ Dentistry Student, Faculty of Dentistry, Zanjan University of Medical Sciences, Zanjan, Iran \\ ${ }^{4}$ Doctor of Dental Surgery (DDS), DMD, Tehran, Iran
}

\begin{abstract}
Summary
Background: Keratocystic odontogenic tumor (KCOT) is a recurrent benign tumor with a keratinized epithelial shape. The treatment methods in KCOT are still debated. The aim of all treatment methods is to eradicate the cyst and to reduce recurrence and surgical complications. This review article was conducted to assess the findings of studies on the diagnosis, management and recurrence of KCOT. Methods: Information were gathered by searching keywords such as management, treatment, pharmacology, surgery and keratocystic odontogenic tumor in international databases such as Web of Science, PubMed and Scopus. The search period was between 2010-2020. Results: Techniques used for the treatment include decompression, marsupialization, enucleation with or without adjunct, Caldwell-Luc surgery and resection. Of the 40 studies, recurrence was observed in 13 studies and the recurrence ranged from 0 to $48 \%$ in different treatment methods. Conclusion: Due to the high recurrence of this disease, it is suggested that long term follow-up be considered after treatment to reduce recurrence. Decision on the treatment should be made considering age, tumor size, and the site of involvement in order to reduce the economic and psychological burden of the disease.
\end{abstract}

\section{Key words}

management - treatment - pharmacology - surgery - keratocystic odontogenic tumor

\section{Souhrn}

Východiska: Keratocystický odontogenní tumor (keratocystic odontogenic tumor - KCOT) je rekurentní benigní tumor tvaru keratinizovaného epitelu. Způsob léčby je stále předmětem diskusí. Cílem všech léčebných metod je eradikace cysty a omezení rekurence a pooperačních komplikací. Tento přehledový článek byl vytvořen s cílem zhodnotit závěry studií zaměěených na diagnostiku, léčbu a rekurenci KCOT. Metody: Informace byly shromažd’ovány po zadání slov management, léčba, farmakologie, operace a keratocystický odontogenní tumor do mezinárodních databází Web of Science, PubMed a Scopus. Sledování dat probíhalo v období let 2010-2020. Výsledky: Mezi techniky používané při léčbě patří dekomprese, marsupializace, enukleace s následnou operací čelistní dutiny ze zevního př́stupu dle Caldwell-Luca nebo bez ní a resekce. Ze 40 studií byla rekurence pozorována v 13 studiích a u různých léčebných metod se pohybovala v rozmezí $0-48 \%$. Závěr: $V$ důsledku vysoké rekurence onemocnění se po léčbě doporučuje dlouhodobé sledování. Rozhodnutí o způsobu léčby by mělo zohledňovat věk pacienta, velikost nádoru a místo výskytu, aby se co nejvíce snížila ekonomická a psychická zátěž tímto onemocněním.

\section{Klíčová slova}

management - léčba - farmakologie - operace - keratocystický odontogenní tumor
The authors declare they have no potential conflicts of interest concerning drugs, products, or services used in the study.

Autor̆i deklarují, že v souvislosti s predmětem studie nemaji žádné komerční zájmy.

The Editorial Board declares that the manuscript met the ICMJE recommendation for biomedical papers.

Redakční rada potvrzuje, že rukopis práce splnil ICMJE kritéria pro publikace zasilané do biomedicínských časopisů

$\square^{\prime \prime}$

Dr Saeed Bashar

Department of Oral and Maxillofa-

cial Surgery

Faculty of Dentistry

Golestan University of Medical

Sciences

Gorgan

Iran

e-mail: basharsaeed61@gmail.com

Submitted/Obdrženo: 15. 4. 2021

Accepted/Přijato: 15. 7. 2021

doi: $10.48095 /$ ccko202210 


\section{Introduction}

Philipsen first coined the term odontogenic keratocyst (OKC) in 1956. [1]. The World Health Organization used the term keratocystic odontogenic tumor (KCOT) for a benign but aggressive tumor of odontogenic origin in 2005. Histologically, KOT is characterized by a thin parakeratinized stratified epithelium [1]. KCOT is a benign neoplasm with a keratinized epithelial outline with a high recurrence rate [2]. KOT is a relatively common developmental odontogenic cyst and represents approximately $10-14 \%$ of all jaw cysts [3]. The reason for the high recurrence rate in KCOT is due to its neoplastic characteristics including high proliferation rate, angiogenesis, presence of daughter cysts and epithelial islands $[4,5]$. Incomplete resection of epithelial structure of KCOT due to the fragility of the tumor tissue is another reason for recurrence $[4,6]$. In radiographic imaging, $\mathrm{KCOT}$ is seen as a unilocular or multilocular well-circumscribed radiolucent lesion with scalloped and corticated margins. Involvement of affected tooth is reported in $25-40 \%$ of cases $[7,8]$. In case of suspicious lesions in mandible or maxilla, CT scan, radionuclide imaging or MRI are used as conjunctive diagnostic methods. CT scan is a better method in identifying bone resorption, osteoporo- sis, periosteal swelling, destruction and calcification [9].

It is believed that keratocysts are originated from dental layer remnants with the following features such as a thin, bandlike lining of stratified squamous epithelium, a corrugated keratinized lining and a spinous cell layer of $8-10$ cells in thickness, a thin, inflammation-free connective tissue capsule, and a lumencontaining varying amounts of desquamated keratin. A predominant parakeratin lining predominates in majority (83-97\%) of KCOT tumors [10,11].

Various treatment options exist for KCOT ranging from conservative managements, including enucleation (with or without curettage), decompression and marsupialization, to aggressive treatments, including enucleation or cryotherapy with liquid nitrogen, and application of Carnoy's and jaw resection. No universal approach has yet been proposed for KCOT and the treatment methods in KCOT are still debated. The aim of all treatment methods is to eradicate the cyst and to reduce recurrence and surgical complications $[12,13]$.

KCOT is commonly asymptomatic and is mainly identified in routine radiographic assessments or panoramic radiographic examinations. Early diagnosis and correct treatment of KCOT is of great importance as surgery and treat- ment of KCOT is complicated and due to its high recurrence rate. Furthermore, there is no comprehensive assessment regarding the superiority of aggressive over conservative management in reducing recurrence. Therefore, this review article was conducted to assess the findings of studies on management and recurrence of KCOT.

\section{Methods}

This study was conducted as a narrative review. Information was gathered by searching keywords such as management, treatment, pharmacology, surgery and keratocystic odontogenic tumor in international databases such as Web of Science, PubMed and Scopus. The search period was between 2010-2020. As the subject was a narrative review, we studied all types of articles. Then the searched articles were evaluated based on the title, method and results. Finally, the related articles were selected for this review.

\section{Results}

In the initial search, 1,500 articles were found. After deleting the duplicate, unrelated or incomplete information, and studies performed in vitro as well as studies without full text, 40 studies were eventually classified as the main study (Tab. 1).

Tab. 1. Main information extracted from the studies articles.

\begin{tabular}{|c|c|c|c|c|c|c|}
\hline Author's name & $\begin{array}{c}\text { No. of } \\
\text { patients }\end{array}$ & Location & Treatment & Recurrence & Follow-up & Conclusion \\
\hline $\begin{array}{l}\text { Agrawal et al } \\
2014 \text { [37] }\end{array}$ & 1 & mandible & $\begin{array}{l}\text { enucleation of the } \\
\text { cyst, peripheral os- } \\
\text { teotomy and use of } \\
\text { Carnoy's solution }\end{array}$ & - & - & $\begin{array}{c}\text { Conservative management using } \\
\text { bismuth iodoform paraffin paste } \\
\text { can be used in cystic lesions, be- } \\
\text { nign lesions, etc.; don't use in case } \\
\text { of malignancies. }\end{array}$ \\
\hline $\begin{array}{l}\text { Borgonovo et al } \\
2011[38]\end{array}$ & 3 & $\begin{array}{l}\text { left ramus } \\
\text { region and } \\
\text { mandible }\end{array}$ & $\begin{array}{l}\text { marsupialization; } \\
\text { after a mean period } \\
\text { of } 10 \text { months, enu- } \\
\text { cleation with periph- } \\
\text { eral ostectomy }\end{array}$ & no & 5 yrs & $\begin{array}{l}\text { The preservation of important an- } \\
\text { atomical structures involved in } \\
\text { the lesion and jaw's continuity is a } \\
\text { main advantage of this technique. }\end{array}$ \\
\hline $\begin{array}{l}\text { Cassoni et al } 2013 \\
\text { [39] }\end{array}$ & 77 & $\begin{array}{l}\text { maxilla } \\
\text { (14 cases) } \\
\text { and } \\
\text { mandible } \\
\text { (63 cases) }\end{array}$ & $\begin{array}{c}\text { enucleation } \\
\text { (55 cases) and } \\
\text { radical resection } \\
\text { (22 cases) ( } 22 \text { cases) }\end{array}$ & $\begin{array}{l}19 \% \text { in enuclea- } \\
\text { tion group and } \\
\text { no recurrence } \\
\text { in resection } \\
\quad \text { group }\end{array}$ & $3-5$ yrs & $\begin{array}{l}\text { Conservative treatment is associ- } \\
\text { ated with high recurrence and re- } \\
\text { section provides the lowest re- } \\
\text { currence rate, but causes most } \\
\text { suffering to the patient. }\end{array}$ \\
\hline
\end{tabular}


Tab. 1 - continuing. Main information extracted from the studies articles.

\begin{tabular}{|c|c|c|c|c|c|c|}
\hline Author's name & $\begin{array}{c}\text { No. of } \\
\text { patients }\end{array}$ & Location & Treatment & Recurrence & Follow-up & Conclusion \\
\hline $\begin{array}{l}\text { Davoodi et al } \\
2013 \text { [40] }\end{array}$ & 1 & mandible & $\begin{array}{l}\text { full surgical ex- } \\
\text { traction, including } \\
\text { the } 2^{\text {nd }} \text { and } 3^{\text {rd }} \text { left } \\
\text { molars }\end{array}$ & no & $6 \mathrm{mos}$ & $\begin{array}{c}\text { A cervical abscess with a traumatic } \\
\text { bone cyst was the result of infected } \\
\text { KCOT. }\end{array}$ \\
\hline $\begin{array}{l}\text { Deboni et al } 2012 \\
\text { [41] }\end{array}$ & 2 & $\begin{array}{l}\text { mandible } \\
\text { body }\end{array}$ & marsupialization & no & $7 \mathrm{yrs}$ & $\begin{array}{l}\text { In young patients, conservative } \\
\text { management with a low rate of } \\
\text { adverse effects should be consid- } \\
\text { ered as treatment choice. }\end{array}$ \\
\hline $\begin{array}{l}\text { de Molon et al } \\
2015 \text { [42] }\end{array}$ & 1 & $\begin{array}{l}\text { left side of } \\
\text { mandible }\end{array}$ & $\begin{array}{l}\text { marsupialization for } \\
\text { lesion decompres- } \\
\text { ssion and conse- } \\
\text { quent lesion size re- } \\
\text { duction; enucleation } \\
\text { for complete KCOT } \\
\text { removal followed } \\
\text { by extraction of } 3^{\text {rd }} \\
\text { mandibular molar }\end{array}$ & no & 5 yrs & $\begin{array}{l}\text { This therapy procedure was a safe } \\
\text { and effective way to control KCOT, } \\
\text { allowing the original lesion to be } \\
\text { reduced while anatomical compo- } \\
\text { nents were preserved. }\end{array}$ \\
\hline $\begin{array}{l}\text { Ebenezer and } \\
\text { Ramalingam } \\
2014 \text { [43] }\end{array}$ & 8 & $\begin{array}{l}\text { mandible } \\
\text { and maxila }\end{array}$ & $\begin{array}{l}\text { resection } \\
\text { ( } 3 \text { cases) and enu- } \\
\text { cleation followed by } \\
\text { application of Car- } \\
\text { noy's solution } \\
\text { ( } 5 \text { cases) }\end{array}$ & no & - & $\begin{array}{l}\text { Treatment modality should be de- } \\
\text { cided on age, extent, aggressive- } \\
\text { ness and nature of the tumour. }\end{array}$ \\
\hline $\begin{array}{l}\text { Güler et al } 2011 \\
\text { [44] }\end{array}$ & 39 & $\begin{array}{c}\text { mandi- } \\
\text { ble }(76.7 \%) \\
\text { and maxilla } \\
(23.3 \%)\end{array}$ & $\begin{array}{c}\text { enucleation } \\
\text { (18 cases), enuclea- } \\
\text { tion with Carnoy's } \\
\text { solution ( } 10 \text { cases); } \\
\text { marsupialization fol- } \\
\text { lowed by enuclea- } \\
\text { tion with Carnoy's } \\
\text { solution ( } 15 \text { cases) }\end{array}$ & no & $\begin{array}{c}40.5 \\
\pm 23.0 \mathrm{mos}\end{array}$ & $\begin{array}{c}\text { Enucleation and Carnoy's } \\
\text { solution in tiny lesions as well as } \\
\text { marsupialization in large lesions } \\
\text { were successful therapeutic } \\
\text { procedures. }\end{array}$ \\
\hline $\begin{array}{l}\text { Gao et al } 2014 \\
{[45]}\end{array}$ & 20 & - & $\begin{array}{l}\text { decompression with } \\
\text { customized thermo- } \\
\text { plastic resin stents }\end{array}$ & no & $1-24$ mos & $\begin{array}{l}\text { Decompression was effective in in- } \\
\text { creasing bone density and reduc- } \\
\text { ing odontogenic cystic lesions of } \\
\text { the jaw, secondary definitive sur- } \\
\text { gery was necessary for aggressive } \\
\text { lesions. }\end{array}$ \\
\hline $\begin{array}{l}\text { Guimarães } \\
\text { et al } 2013 \text { [46] }\end{array}$ & 3 & $\begin{array}{l}\text { left mandib- } \\
\text { ular ramus, } \\
\text { right mandib- } \\
\text { ular angle re- } \\
\text { gion, right } \\
\text { mandibular } \\
\text { angle }\end{array}$ & surgical resection & no & $\begin{array}{l}4 \mathrm{yrs} \\
6 \mathrm{mos} \\
9 \mathrm{mos}\end{array}$ & $\begin{array}{l}\text { Understanding both biological } \\
\text { and molecular levels could lead to } \\
\text { therapy and prognosis guidelines } \\
\text { for people with this condition. }\end{array}$ \\
\hline $\begin{array}{l}\text { Hasheminia et al } \\
2014 \text { [47] }\end{array}$ & 1 & $\begin{array}{l}\text { mandibu- } \\
\text { lar body and } \\
\text { maxilla }\end{array}$ & marsupialization & no & $30 \mathrm{mos}$ & $\begin{array}{l}\text { Marsupialization with or without } \\
\text { adjunctive treatments is a conserv- } \\
\text { ative and effective protocol with } \\
\text { low morbidity and cost. }\end{array}$ \\
\hline $\begin{array}{l}\text { Kebede et al } 2016 \\
\text { [48] }\end{array}$ & 1 & $\begin{array}{l}\text { right mandib- } \\
\text { ular angle to } \\
\text { left mandibu- } \\
\text { lar ramus }\end{array}$ & $\begin{array}{c}\text { total } \\
\text { mandibulectomy }\end{array}$ & no & $6 \mathrm{mos}$ & $\begin{array}{l}\text { Occurrence of KCOT should be con- } \\
\text { sidered at the time of clinical diag- } \\
\text { nosis, although it is rare. }\end{array}$ \\
\hline
\end{tabular}


Tab. 1 - continuing. Main information extracted from the studies articles.

\begin{tabular}{|c|c|c|c|c|c|c|}
\hline Author's name & $\begin{array}{l}\text { No. of } \\
\text { patients }\end{array}$ & Location & Treatment & Recurrence & Follow-up & Conclusion \\
\hline $\begin{array}{l}\text { Kim et al } 2017 \\
\text { [49] }\end{array}$ & 1 & $\begin{array}{l}\text { mandibular } \\
\text { left primary } \\
\text { molar }\end{array}$ & $\begin{array}{l}\text { marsupialization, de- } \\
\text { compression after } \\
2 \text { yrs, curettage after } \\
1 \text { yr, decompression } \\
\text { repeated after } 1 \mathrm{yr}\end{array}$ & $\begin{array}{c}\text { yes } \\
(2,3 \text { and } 4 \\
\text { yrs after first } \\
\text { surgery })\end{array}$ & 4 yrs & $\begin{array}{l}\text { Initial decompression and later pe- } \\
\text { ripheral ostectomy can be a good } \\
\text { conservative treatment option for } \\
\text { young patients. }\end{array}$ \\
\hline $\begin{array}{l}\text { Koçak- } \\
\text {-Berberoğlu } \\
\text { et al } 2012 \text { [50] }\end{array}$ & 4 & $\begin{array}{l}\text { maxilla } \\
\text { (3 cases), } \\
\text { maxilla and } \\
\text { mandible } \\
(1 \text { case })\end{array}$ & enucleation & - & - & $\begin{array}{l}\text { Lesions in the maxillofacial region } \\
\text { could be better shown in the cor- } \\
\text { rect dimensions by cone beam } \\
\text { computed tomography vs. pano- } \\
\text { ramic radiograph. }\end{array}$ \\
\hline $\begin{array}{l}\text { Kunihiro et al } \\
2014 \text { [51] }\end{array}$ & 1 & maxilla & $\begin{array}{l}\text { enucleation, the } \\
\text { whole tumor was } \\
\text { successfully re- } \\
\text { moved using a bidi- } \\
\text { rectional approach } \\
\text { from the fistula and } \\
\text { from the antros- } \\
\text { tomy in the middle } \\
\text { meatus }\end{array}$ & no & $6 \mathrm{mos}$ & $\begin{array}{c}\text { Collaboration between an oral sur- } \\
\text { geon and an otorhinolaryngologist } \\
\text { could provide a minimally invasive } \\
\text { and also radical surgical treatment } \\
\text { for certain kinds of tumor in the } \\
\text { head and neck region. }\end{array}$ \\
\hline $\begin{array}{l}\text { Lacarbonara et al } \\
2014 \text { [52] }\end{array}$ & 1 & $\begin{array}{l}\text { upper left } \\
\text { maxillary } \\
\text { region }\end{array}$ & $\begin{array}{l}\text { surgical antral } \\
\text { cystectomy with } \\
\text { the Caldwell-Luc } \\
\text { method to remove } \\
\text { the lesion and } \\
\text { infected sinus } \\
\text { mucosa }\end{array}$ & no & 24 mons & $\begin{array}{l}\text { To create a proper therapeutic } \\
\text { protocol and achieve a favorable } \\
\text { prognosis for the management } \\
\text { of this patient, interdisciplinary } \\
\text { collaboration of different } \\
\text { specialities and careful preparation } \\
\text { are essential. }\end{array}$ \\
\hline $\begin{array}{l}\text { Ledderhof et al } \\
2017 \text { [53] }\end{array}$ & 32 & $\begin{array}{l}\text { mandible } \\
(27 \text { cases }) \\
\text { and maxilla } \\
\text { ( } 5 \text { cases })\end{array}$ & $\begin{array}{l}\text { topical application } \\
\text { of } 5 \% 5 \text {-FU } \\
\text { (11 cases) or mod- } \\
\text { ified Carnoy's solu- } \\
\text { tion ( } 21 \text { cases) after } \\
\text { enucleation and pe- } \\
\text { ripheral ostectomy }\end{array}$ & $\begin{array}{l}\text { no (in 5-FU } \\
\text { group), yes } \\
\text { (4 recurrences } \\
\text { in Carnoy's so- } \\
\text { lution group) }\end{array}$ & $\begin{array}{l}41.3 \pm 3.8 \\
\text { mos and } \\
35.0 \pm 8.5 \\
\text { mos }\end{array}$ & $\begin{array}{l}\text { Topical 5-FU is a new medication } \\
\text { for KOTs that takes a molecularly } \\
\text { targeted approach to treatment. }\end{array}$ \\
\hline $\begin{array}{l}\text { Leung et al } 2016 \\
\text { [24] }\end{array}$ & 105 & $\begin{array}{l}\text { mandible } \\
\text { (83 cases) } \\
\text { and maxilla } \\
\text { (22 cases) }\end{array}$ & $\begin{array}{l}\text { enucleation and ap- } \\
\text { plication of Carnoy's } \\
\text { solution, assessment } \\
\text { of surgical morbidi- } \\
\text { ties associated with } \\
\text { this treatment }\end{array}$ & $11.4 \%$ & 24-313 mos & $\begin{array}{l}\text { Enucleation and application of Car- } \\
\text { noy's solution for the treatment of } \\
\text { KCOTs leads to relatively low recur- } \\
\text { rence and surgical morbidity rates. }\end{array}$ \\
\hline Liu et al 2012 [54] & 1 & $\begin{array}{c}\text { right } \\
\text { mandible }\end{array}$ & $\begin{array}{c}\text { segmental } \\
\text { mandibulectomy }\end{array}$ & yes & 14 yrs & $\begin{array}{l}\text { When a lesion occurs in the } \\
\text { ascending ramus of the mandible, } \\
\text { particularly when the cortical bone } \\
\text { is perforated, KCOT may reoccur in } \\
\text { the surrounding soft } \\
\text { tissue. }\end{array}$ \\
\hline $\begin{array}{l}\text { Zhao et al } 2012 \\
\text { [55] }\end{array}$ & 19 & $\begin{array}{l}\text { mandible } \\
\text { (15 cases), } \\
\text { maxilla } \\
\text { ( } 4 \text { cases })\end{array}$ & $\begin{array}{l}\text { enucleation } \\
\text { (12 cases) or enucle- } \\
\text { ation with Carnoy's } \\
\text { solution ( } 7 \text { cases) }\end{array}$ & $\begin{array}{l}\text { yes }(15 \text { out of } \\
19(78.9 \%) \text { re- } \\
\text { curred within } \\
6 \text { yrs, while } \\
4(21.1 \%) \text { re- } \\
\text { curred after } \\
6 \text { yrs })\end{array}$ & $6 \mathrm{yrs}$ & $\begin{array}{l}\text { The recurrent KCOTs had a ten- } \\
\text { dency to be more unilocular or } \\
\text { multilocular than the primary } \\
\text { cases, with a unilocular-to-multiloc- } \\
\text { ular ratio of } 1.1: 1 .\end{array}$ \\
\hline
\end{tabular}


Tab. 1 - continuing. Main information extracted from the studies articles.

\begin{tabular}{|c|c|c|c|c|c|c|}
\hline Author's name & $\begin{array}{c}\text { No. of } \\
\text { patients }\end{array}$ & Location & Treatment & Recurrence & Follow-up & Conclusion \\
\hline $\begin{array}{l}\text { Morais de Melo } \\
\text { et al } 2012 \text { [56] }\end{array}$ & 1 & mandible & $\begin{array}{l}\text { decompression; } \\
\text { after } 6 \text { mos the le- } \\
\text { sion cyst was lower } \\
\text { than before decom- } \\
\text { pression surgery; } \\
\text { enucleation and cu- } \\
\text { rettage of the cyst }\end{array}$ & no & 3 years & $\begin{array}{c}\text { The success rate of decompression } \\
\text { is as high as that of an aggressive } \\
\text { treatment. }\end{array}$ \\
\hline $\begin{array}{l}\text { Motwani et al } \\
2011 \text { [57] }\end{array}$ & 2 & $\begin{array}{l}\text { maxilla, right } \\
\text { mandible }\end{array}$ & $\begin{array}{l}\text { both our cases were } \\
\text { treated by enuclea- } \\
\text { tion along with ex- } \\
\text { traction of associ- } \\
\quad \text { ated teeth }\end{array}$ & no & $1 \mathrm{yr}$ & $\begin{array}{c}\text { Considering the high recurrence } \\
\text { rate of these lesions, post-opera- } \\
\text { tive follow-up of at least } 5 \text { years is } \\
\text { necessary. }\end{array}$ \\
\hline $\begin{array}{l}\text { Naruse et al } 2017 \\
\text { [58] }\end{array}$ & 63 & $\begin{array}{l}\text { mandible } \\
\text { (44 cases), } \\
\text { maxilla } \\
(18 \text { cases }), \\
\text { mandible } \\
\text { and maxilla } \\
(1 \text { case })\end{array}$ & $\begin{array}{c}\text { conservative } \\
\text { (37 cases including } \\
29 \text { cases of no treat- } \\
\text { ment and } 8 \text { cases } \\
\text { of apicoectomy), } \\
\text { radical treatment } \\
\text { ( } 22 \text { extractions and } \\
6 \text { cases with no } \\
\text { contact with the } \\
\text { root) }\end{array}$ & yes (3 tumors) & - & $\begin{array}{l}\text { Conservative treatment was the } \\
\text { biggest independent risk factor for } \\
\text { tumor recurrence. }\end{array}$ \\
\hline $\begin{array}{l}\text { Nomura et al } \\
2015 \text { [59] }\end{array}$ & 1 & maxilla & $\begin{array}{l}\text { endoscopic modi- } \\
\text { fied medial maxillec- } \\
\text { tomy technique }\end{array}$ & no & 1 year & $\begin{array}{l}\text { Endoscopic modified medial max- } \\
\text { illectomy is a minimally invasive } \\
\text { procedure that allows for a direct } \\
\text { field of vision for maxillary KCOT } \\
\text { treatment. }\end{array}$ \\
\hline Ohki 2012 [60] & 1 & $\begin{array}{l}\text { right maxil- } \\
\text { lary sinus }\end{array}$ & $\begin{array}{l}\text { endoscopic sinus } \\
\text { surgery to widen the } \\
\text { maxillary ostium } \\
\text { and remove } \\
\text { a piece of the } \\
\text { cystic wall } \\
\text { during transnasal } \\
\text { marsupialization }\end{array}$ & no & 1 year & $\begin{array}{l}\text { Reductive change and minimal sur- } \\
\text { gical invasion, making it advan- } \\
\text { tageous in the case of secondary } \\
\text { surgery, for full removal with less } \\
\text { problems in the bones and sur- } \\
\text { rounding tissue. }\end{array}$ \\
\hline $\begin{array}{l}\text { Ribeiro Jr. et al } \\
2012 \text { [61] }\end{array}$ & 22 & $\begin{array}{l}\text { mandible } \\
\text { (16 cases), } \\
\text { maxilla } \\
\text { (6 cases) }\end{array}$ & $\begin{array}{l}\text { Carnoy's solution } \\
\text { combined with } \\
\text { peripheral ostectomy }\end{array}$ & $4.5 \%$ & $\begin{array}{l}\text { mean of } \\
42.9 \text { mos }\end{array}$ & $\begin{array}{l}\text { KOTs can be effectively treated with } \\
\text { Carnoy's solution and peripheral } \\
\text { ostectomy. }\end{array}$ \\
\hline $\begin{array}{l}\text { Roopak et al } 2013 \\
\text { [62] }\end{array}$ & 3 & mandible & $\begin{array}{l}\text { decompression and } \\
\text { marsupialization to } \\
\text { avoid secondary de- } \\
\text { formity ( } 1 \text { case), enu- } \\
\text { cleation with exci- } \\
\text { sion of overlying } \\
\text { mucosa and chemi- } \\
\text { cal cauterization car- } \\
\text { ried out using Car- } \\
\text { noy's solution } \\
\text { (1 case), enucleation } \\
\text { and peripheral } \\
\text { osteotomy ( } 1 \text { case) }\end{array}$ & no & $\begin{array}{l}3 \mathrm{mos}, 1 \mathrm{yr} \\
2 \mathrm{yrs}\end{array}$ & $\begin{array}{l}\text { The priority of the treatment } \\
\text { method chosen should depend on } \\
\text { the morbidity, size of the lesion, re- } \\
\text { currence rate and patients' qual- } \\
\text { ity of life. Large cysts can be suc- } \\
\text { cessfully treated with conservative } \\
\text { approach. }\end{array}$ \\
\hline
\end{tabular}


Tab. 1 - continuing. Main information extracted from the studies articles.

\begin{tabular}{|c|c|c|c|c|c|c|}
\hline Author's name & $\begin{array}{l}\text { No. of } \\
\text { patients }\end{array}$ & Location & Treatment & Recurrence & Follow-up & Conclusion \\
\hline $\begin{array}{l}\text { Scartezini et al } \\
2012[63]\end{array}$ & 1 & $\begin{array}{l}\text { left mandibu- } \\
\text { lar ramus }\end{array}$ & $\begin{array}{l}\text { surgery involved os- } \\
\text { teotomy, detach- } \\
\text { ment of cystic lesion } \\
\text { and removal of } 36^{\text {th }} \text {, } \\
37^{\text {th }} \text { and } 38^{\text {th }} \text { teeth }\end{array}$ & no & $12 \mathrm{mos}$ & $\begin{array}{l}\text { Accurate radiographic, micro- } \\
\text { scopic and clinical examinations } \\
\text { are essential to choose the most } \\
\text { effective therapy and definitive } \\
\text { diagnosis. }\end{array}$ \\
\hline $\begin{array}{l}\text { Schussel et al } \\
2012 \text { [64] }\end{array}$ & 25 & $\begin{array}{c}\text { mandible } \\
\text { (20 cases), } \\
\text { maxilla } \\
\text { (4 cases), } \\
\text { mandible } \\
\text { and maxilla } \\
\text { (1 case) }\end{array}$ & $\begin{array}{l}\text { surgical treatment } \\
\text { associated or not } \\
\text { with adjuvant ther- } \\
\text { apy, i.e. cryother- } \\
\text { apy and Carnoy's } \\
\text { solution }\end{array}$ & $48 \%$ & $\begin{array}{c}\text { mean of } 18 \\
\text { mos }\end{array}$ & $\begin{array}{l}\text { Previous diagnosis before enuclea- } \\
\text { tion procedure and long-term fol- } \\
\text { low-up for recurrence early detec- } \\
\text { tion is important. }\end{array}$ \\
\hline $\begin{array}{l}\text { Bharani et al } 2012 \\
\text { [65] }\end{array}$ & 1 & $\begin{array}{c}\text { right } \\
\text { mandibular } \\
\text { posterior } \\
\text { region, left } \\
\text { hemi-maxilla } \\
\end{array}$ & $\begin{array}{l}\text { a transoral approach, } \\
\text { enucleation of man- } \\
\text { dibular cyst, followed } \\
\text { by application of } \\
\text { Cornoy's solution; ex- } \\
\text { traction of affected } \\
\text { teeth; treatment of } \\
\text { maxillary cyst by sim- } \\
\text { ilar method }\end{array}$ & no & $\begin{array}{l}2 \text { yrs }-10 \\
\text { mos }\end{array}$ & - \\
\hline $\begin{array}{l}\text { Srivatsan et al } \\
2014 \text { [66] }\end{array}$ & 2 & mandible & $\begin{array}{l}\text { marsupialization by } \\
\text { excision of the over- } \\
\text { lying mucosa and } \\
\text { opening of appropri- } \\
\text { ately sized window }\end{array}$ & no & $2 \mathrm{yrs}$ & $\begin{array}{l}\text { The marsupialization approach } \\
\text { resulted in the complete clearance } \\
\text { of the lesion, eliminating the need } \\
\text { for further definitive cystectomy } \\
\text { treatment }\end{array}$ \\
\hline $\begin{array}{l}\text { Sivanmalai et al } \\
2012 \text { [67] }\end{array}$ & 1 & mandible & $\begin{array}{l}\text { enucleation, periph- } \\
\text { eral ostectomy, fol- } \\
\text { lowed by single ap- } \\
\text { plication of Carnoy's } \\
\text { solution, including } \\
\text { over the inferior al- } \\
\text { veolar plexus }\end{array}$ & no & $36 \mathrm{mos}$ & $\begin{array}{c}\text { Carnoy's solution is a supplemen- } \\
\text { tary treatment for keratocystic od- } \\
\text { ontogenic tumors applied to the } \\
\text { inferior alveolar vascular-nervous } \\
\text { plexus. }\end{array}$ \\
\hline $\begin{array}{l}\text { Vázquez-Romero } \\
\text { et al } 2016 \text { [68] }\end{array}$ & 1 & maxilla & $\begin{array}{l}\text { curettage of the } \\
\text { bone tissue to } \\
\text { remove the cyst }\end{array}$ & no & $6 \mathrm{mos}$ & - \\
\hline $\begin{array}{l}\text { Warburton et al } \\
2014 \text { [69] }\end{array}$ & 80 & $\begin{array}{l}\text { mandible } \\
\text { and maxilla }\end{array}$ & $\begin{array}{l}12 \text { patients treated } \\
\text { by resection and } \\
68 \text { patients } \\
\text { managed by } \\
\text { enucleation and cu- } \\
\text { rettage, enucleation } \\
\text { with peripheral os- } \\
\text { tectomy, or decom- } \\
\text { pression with sec- } \\
\text { ondary enucleation }\end{array}$ & yes & 2-168 mos & $\begin{array}{l}\text { The involvement of the pterygoid } \\
\text { muscles was the most common } \\
\text { reason for KCOT excision, but } \\
\text { malignant alteration was also } \\
\text { a factor. }\end{array}$ \\
\hline $\begin{array}{l}\text { Zhou et al } 2014 \\
\text { [70] }\end{array}$ & 1 & maxilla & $\begin{array}{l}\text { modified treatment } \\
\text { with enucleation, } \\
\text { grinding and cryo- } \\
\text { therapy were } \\
\text { effective methods of } \\
\text { treatment for KCOT }\end{array}$ & no & $8 \mathrm{mos}$ & $\begin{array}{l}\text { A suitable treatment option for } \\
\text { giant KCOTs may be modified. }\end{array}$ \\
\hline
\end{tabular}


Tab. 1 - continuing. Main information extracted from the studies articles.

\begin{tabular}{|c|c|c|c|c|c|c|}
\hline Author's name & $\begin{array}{c}\text { No. of } \\
\text { patients }\end{array}$ & Location & Treatment & Recurrence & Follow-up & Conclusion \\
\hline $\begin{array}{l}\text { Yang et al } 2011 \\
\text { [71] }\end{array}$ & $\begin{array}{c}181 \\
(220 \\
\text { KCOTs })\end{array}$ & $\begin{array}{l}\text { mandible } \\
\text { (153 cases), } \\
\text { maxilla } \\
\text { (67 cases) }\end{array}$ & $\begin{array}{c}\text { enucleation or cu- } \\
\text { rettage ( } 164 \text { cases), } \\
\text { marsupialization } \\
\text { or decompression } \\
\text { (17 cases), lateral } \\
\text { decortication } \\
\text { (13 cases) }\end{array}$ & $10.78 \%$ & $5 \mathrm{yrs}$ & $\begin{array}{l}\text { The most common treatment } \\
\text { method was enucleation } \\
\text { or curettage, followed by } \\
\text { marsupialization or decompression } \\
\text { and lateral decortication. }\end{array}$ \\
\hline $\begin{array}{l}\text { Yildirim et al } \\
2010 \text { [72] }\end{array}$ & 3 & $\begin{array}{l}\text { mandible } \\
\text { ( } 2 \text { cases), } \\
\text { maxilla } \\
(1 \text { case })\end{array}$ & $\begin{array}{l}\text { conservative treat- } \\
\text { ment (enucleation } \\
\text { followed by open } \\
\text { packing) }\end{array}$ & no & $2 \mathrm{yrs}$ & $\begin{array}{l}\text { This technique could be a possible } \\
\text { choice with a view of offering low } \\
\text { morbidity rate and low recurrence } \\
\text { rate, particularly in young patients. }\end{array}$ \\
\hline $\begin{array}{l}\text { Jafaripozve et al } \\
2013 \text { [73] }\end{array}$ & 1 & $\begin{array}{l}\text { right side of } \\
\text { the mandi- } \\
\text { ble at molar } \\
\text { region }\end{array}$ & $\begin{array}{l}\text { surgical treatment } \\
\text { by enucleation and } \\
\text { curettage } 5 \text { years } \\
\text { ago, resection }\end{array}$ & yes & $5 \mathrm{yrs}$ & $\begin{array}{l}\text { The patient's rate of recurrence and } \\
\text { morbidity is reduced when the op- } \\
\text { timum treatment method is chosen } \\
\text { and followed up on on a regular } \\
\text { basis throughout his or her life. }\end{array}$ \\
\hline $\begin{array}{l}\text { Ribeiro-Júnior } \\
\text { et al } 2017 \text { [74] }\end{array}$ & 40 & $\begin{array}{l}\text { mandible } \\
\text { ( } 27 \text { cases), } \\
\text { maxilla } \\
\text { (13 cases) }\end{array}$ & $\begin{array}{l}27 \text { lesions treated } \\
\text { by enucleation } \\
\text { and } 13 \text { underwent } \\
\text { decompresssion }\end{array}$ & $15 \%$ & 12-102 mos & $\begin{array}{l}\text { Significant difference for the recur- } \\
\text { rence of lesions was not shown; } \\
\text { this could be due to the effec- } \\
\text { tiveness of the complementary } \\
\text { treatments. }\end{array}$ \\
\hline $\begin{array}{l}\text { Oliveira et al } 2017 \\
\text { [75] }\end{array}$ & 1 & mandible & $\begin{array}{l}\text { decompresssion tech- } \\
\text { nique (during } 8 \text { mos), } \\
\text { recurrence in mandi- } \\
\text { bular ramus in a fol- } \\
\text { low-up of } 1 \text { year and } \\
6 \text { months, enucleation } \\
\text { and curettage associ- } \\
\text { ated with cryotherapy }\end{array}$ & $\begin{array}{l}\text { no recurrence } \\
3 \text { years later }\end{array}$ & $3 \mathrm{yrs}$ & - \\
\hline
\end{tabular}

5-FU - 5-fluorouracil, KCOT - keratocystic odontogenic tumor, mo(s) - month(s), yr(s) - year(s)

In these studies, the sample size varied from 1 person to 181 people. The study showed that the site of KCOT was mostly in the mandible. Techniques used for treatment included decompression, marsupialization, enucleation with or without adjunct (Carnoy's solution, 5-fluorouracil (5-FU)), Caldwell-Luc surgery and resection, for example mandibulotomy, antrostomy, endoscopic modified medial maxillectomy (EMMM), etc. Of the 40 studies, recurrence was observed in 13 studies and the recurrence ranged from 0 to $48 \%$ in different treatment methods.

\section{Discussion}

The aim of this review article was to assess the effective management methods in KCOT. A total of 40 original articles were reviewed. Majority of the articles were case reports. The most common affected site was mandible. The sample size varied from one to 181 subjects in different studies. The difference in sample size made the interpretation of the findings difficult.

Review of the studies indicated that the management methods used for KCOT comprised of various surgical approaches, including decompression, marsupialization, enucleation with or without adjunct (Carnoy's solution, 5-FU), Caldwell-Luc surgery and resection, for example mandibulotomy, antrostomy, EMMM, etc.

\section{Decompression}

Decompression is defined as any technique that reduces the pressure inside the cyst. Increased pressure inside the cyst results in the growth and expansion of the cyst [14]. Decompression is considered as an alternative and a more conservative approach that annihilates the predisposing factors for tumor expansion by continues drainage of the cyst [15].

Decompression minimizes adjacent tissue injury. However the effects of decompression on prevention of recurrence is yet to be discussed $[16,17]$. The important superiority of marsupialization over decompression is preserving the important anatomical structures including inferior alveolar nerve and preventing following deformities [18].

\section{Marsupialization}

Marsupialization was first described by Partch in 1892 [19]. This approach in- 
cludes incision of a part of the body of KCOT tumor and suturing the borders in adjacent mucus. The resultant surgical window opens the cyst in oral cavity. In decompression technique, a drain is placed inside the lesion that connects the cyst to the oral cavity. This will reduce intracystic pressure and causes bone formation [20]. The difference between decompression and marsupialization is in the use of a cylindrical device (drain) for preventing mucosal closure [21]. Based on the findings of the study by Tabrizi et al, the recurrence rate might be lower in decompression compared to marsupialization [22].

\section{Enucleation with and without adjuncts}

Enucleate refers to the removal of a tumor's envelope in its entirety. Curettage is the process of removing growths or other material from the cavity's wall. This technique has been used as a treatment approach for KCOT for many years. Although enucleation or curettage are superior to marsupialization in providing adequate sample for tissue analysis, but the reported recurrence rate $(62.5 \%)$ is not considered desirable for a treatment approach. Some studies combined enucleation or curettage with adjuvant therapy including chemical solutions (Carnoy's) or cryosurgical agents (liquid nitrogen) for the treatment of KCOT $[23,24]$. Similarly, a study reported a significant effect for combined enucleation with 5-FU in the treatment of KCOT, with fewer post-operative complications and recurrence compared to modified Carnoy's solution.

\section{Enucleation with Carnoy's solution}

Carnoy's solution was first used for the treatment of cystic lesions and fistulae by Cutler and Zollinger [25]. Later some studies reported the use of Carnoy's solution in the treatment of unicystic ameloblastoma and ossifying fibroma. Actually, of the difficulty of enucleating the friable and thin wall of the KCOT as one piece, and due to the small satellite cysts, consequently, treatment should be targeted to eliminate the possible vital cells left behind in the defect. This is due to the use of a light, non-penetrating cauterizing agent such as Carnoy's solution ( $3 \mathrm{~mL}$ chloroform, $6 \mathrm{~mL}$ pure ethanol, $1 \mathrm{~mL}$ glacial acetic acid, and $1 \mathrm{~g}$ ferric chloride) [26]. Furthermore, Carnoy's solution might penetrate cancellous spaces and deviate or fix the remaining tumor [27]. Currently the reformulated Carnoy's solution, without chloroform, is being used as exposure to chloroform may result in cancer or affect fertility [28]. Electrocauterization has been used to prevent recurrence in cases where KCOT invades buccal or lingual cortex [29].

Various studies and evaluations have pointed out to the high efficacy of the administration of Carnoy's solution in combination with enucleation. The use of Carnoy's solution during surgical treatment of invasive cystic lesions reduced the recurrence risk from $6-80 \%$ to $6.6 \%$ [24,30-32]. Furthermore, some studies used Carnoy's solution as an adjuvant therapy after peripheral osteotomy, which reduced recurrence rate [33]. Güler et al also suggested to use this technique in small unilocular lesions [22].

\section{Resection with or without preservation of the continuity of the jaw}

Segmental resection refers to the surgical removal of a segment of the mandible or maxilla without retaining bone continuity, while marginal resection refers to the surgical excision of a lesion intact with a rim of uninvolved bone while keeping bone continuity [24,34].

Resection technique is used in KCOT cases with very large lesions with pterygoid muscles involvement, malignant changes or frequent recurrences. Another indication for resection is perforation of bone cortex and involvement of soft tissue with the probability of vital structure involvement including lateral skull base and orbit [34].

Although some studies reported that the recurrence rate after resection was zero [34,35], but resection is considered as an extreme method as it results in significant complications and requires reconstruction measures for the restoration of functional and aesthetic purposes. This will add the psychological and economic burden of the disease and may reduce the quality of life in KCOT patients at all age groups, especially in the youth.

KCOT tumors have high recurrence rate $[31,36]$. The findings of this review indicated that the recurrence ranged from 0 to $48 \%$ in different treatment methods. Therefore, it is suggested that long term follow-up should be considered after treatment to reduce recurrence. This review also found that the recurrence rate was higher in conservative treatments compared to aggressive treatments; therefore, it is suggested that the treatment method should be decided carefully. Decision on the treatment should be made considering age, tumor size, and the site of involvement in order to reduce the economic and psychological burden of the disease.

\section{Conclusion}

Current developments in genetic and molecular techniques have increased our knowledge about KCOT and resulted in new treatment choices. Due to the high recurrence of this disease, it is suggested that long term follow-up be considered after treatment to reduce recurrence. Also it is recommended that the treatment method be selected carefully. We suggest that physicians should consider age, tumor size and other factors in choosing the treatment option in order to prevent recurrence. According to the studies reviewed, the use of enucleation and Carnoy's solution for small lesions, marsupialization and decompressing for larger lesions and resection for very large lesions is suggested. As KCOT is more common in the second decade of life, long term follow-up is recommended.

\section{Data availability \\ All generated data were used in this study.}

\section{References}

1. Philipsen HP. Keratocystic odontogenic tumour. In: Barnes L, Eveson J, Reichart P et al (eds). Pathology and genetics of head and neck tumours, WHO classification of tumours. Lyon: IARC Press 2005: 306-307.

2. Chemli H, Dhouib M, Karray F et al. Risk factors for recurrence of maxillary odontogenic keratocysts. Rev Stomatol Chir Maxillofac 2010; 111(4): 189-192. doi: 10.1016/j. stomax.2009.07.011. 
3. Neville BW, Damm DD, Allen CM et al. Color atlas of ora and maxillofacial diseases. Elsevier Health Sciences 2018. 4. Mendes RA, Carvalho JFC, van der Waal I. Characterization and management of the keratocystic odontogenic tumor in relation to its histopathological and biological features. Oral Oncol 2010; 46(4): 219-225. doi: 10.1016/j. oraloncology.2010.01.012

5. Suemitsu M. A pathomorphological study of fractal analysis in parenchymal-stromal border on keratocystic odontogenic tumor - with special reference to proliferative activity and vascular distribution. Int J Oral Med Sc 2012; 10(4): 372-383. doi: 10.5466/ijoms.10.372

6. Okamoto E, Kikuchi K, Miyazaki Y et al. Significance of podoplanin expression in keratocystic odontogenic tumor. J Oral Pathol Med 2010; 39(1): 110-114. doi 10.1111/j.1600-0714.2009.00851.x

7. Buckley PC, Seldin EB, Dodson TB et al. Multilocularity as a radiographic marker of the keratocystic odontogenic tumor. J Oral Maxillofac Surg 2012; 70(2): 320-324. doi: 10.1016/j.joms.2011.03.012

8. Neville B, Damm DD, Allen C. Oral and maxillofacial pathology. Philadelphia 2002

9. Kaneda T, Minami M, Kurabayashi T. Benign odontogenic tumors of the mandible and maxilla. Neuroimaging Clin N Am 2003; 13(3): 495-507. doi: 10.1016/s1052 5149(03)00032-7.

10. Kakarantza-Angelopoulou E, Nicolatou O. Odontogenic keratocysts: clinicopathologic study of 87 cases. J Oral Maxillofac Surg 1990; 48(6): 593-599. doi: 10.1016/s0278-2391(10)80472-0.

11. Brannon RB. The odontogenic keratocyst: a clinicopathologic study of 312 cases. Part II. Histologic features. Oral Surg Oral Med Oral Pathol 1977; 43(2): 233-255. doi 10.1016/0030-4220(77)90161-x

12. Kolokythas A, Fernandes RP, Pazoki A et al. Odontogenic keratocyst: to decompress or not to decompress? A comparative study of decompression and enucleation versus resection/peripheral ostectomy. J Oral Maxillofac Surg 2007; 65(4): 640-644. doi: 10.1016/j. joms.2006.06.284

13. Tolstunov L, Treasure T. Surgical treatment algorithm for odontogenic keratocyst: combined treatment of odontogenic keratocyst and mandibular defect with marsupialization, enucleation, iliac crest bone graft, and dental implants. J Oral Maxillofac Surg 2008; 66(5): 1025-1036. doi: 10.1016/j.joms.2007.08.014

14. Pogrel MA. Treatment of keratocysts: the case for decompression and marsupialization J Oral Maxillofac Surg 2005; 63(11): 1667-1673. doi: 10.1016/j.joms.2005.08.008. 15. Stoor P, Apajalahti S, Kontio R. Regeneration of cystic bone cavities and bone defects with bioactive glass S53P4 in the upper and lower jaws. J Craniofac Surg 2017; 28(5): 1197-1205. doi: 10.1097/SCS.0000000000003 649.

16. Al-Moraissi EA, Pogrel MA, Ellis E 3rd. Enucleation with or without adjuvant therapy versus marsupialization with or without secondary enucleation in the treatment of keratocystic odontogenic tumors: a systematic review and meta-analysis. J Craniomaxillofac Surg 2016; 44(9): 1395-1403. doi: 10.1016/.j.jcms.2016.05.020

17. Pogrel MA. Decompression and marsupialization as definitive treatment for keratocysts - a partial retraction. J Oral Maxillofac Surg 2007; 65(2): 362-363. doi: 10.1016/j. joms.2006.09.032.

18. Wushou A, Zhao YJ, Shao ZM. Marsupialization is the optimal treatment approach for keratocystic odonto genic tumour. J Craniomaxillofac Surg 2014; 42(7): 1540 1544. doi: 10.1016/j.jcms.2014.04.027.

19. Pogrel MA. Treatment of keratocysts: the case for decompression and marsupialization. J Oral Maxillofac Surg 2005; 63(11): 1667-1673. doi: 10.1016/j.joms.2005.08.008. 20. de Castro MS, Caixeta CA, de Carli ML et al. Conservative surgical treatments for nonsyndromic odontogenic keratocysts: a systematic review and meta-analysis. Clin
Oral Investig 2018; 22(5): 2089-2101. doi: 10.1007/s00784017-2315-8.

21. Maurette PE, Jorge J, de Moraes M. Conservative treat ment protocol of odontogenic keratocyst: a preliminary study. J Oral Maxillofac Surg 2006; 64(3): 379-383. doi: 10.1016/j.joms.2005.11.007.

22. Tabrizi R, Hosseini Kordkheili MR, Jafarian M et al. Decompression or marsupialization; which conservative treatment is associated with low recurrence rate in keratocystic odontogenic tumors? A systematic review. Jent (Shiraz) 2019: 20(3): 145-151. doi: 10.30476/DENTJODS.2019.44899.

23. Jensen J, Sindet-Pedersen S, Simonsen EK. A comparative study of treatment of keratocysts by enucleation or enucleation combined with cryotherapy. A preliminary report. J Craniomaxillofac Surg 1988; 16(8): 362-365. doi: 10.1016/s1010-5182(88)80080-5.

24. Leung YY, Lau SL, Tsoi KY et al. Results of the treatment of keratocystic odontogenic tumours using enucleation and treatment of the residual bony defect with Carnoy's solution. Int J Oral Maxillofac Surg 2016; 45(9): 1154-1158. doi: 10.1016/j.ijom.2016.02.002.

25. Cutler EC, Zollinger R. The use of sclerosing solutions in the treatment of cysts and fistulae. Am J Surg 1933; 19(3): 411-418. doi: 10.1016/50002-9610(33)90796-5. 26. Morgan TA, Burton CC, Qian F. A retrospective review of treatment of the odontogenic keratocyst. J Ora Maxillofac Surg 2005; 63(5): 635-639. doi: 10.1016/j. joms.2004.07.026.

27. Voorsmit RA, Stoelinga PJ, van Haelst UJ. The management of keratocysts. J Maxillofac Surg 1981; 9(4): 228-236. doi: 10.1016/s0301-0503(81)80049-5.

28. Madhulaxmi M, Wahab PU. Carnoy's solution as a surgical medicament in the treatment of keratocystic odontogenic tumour. Int J Pharm Bio Sci 2014: 5(1): B492-B495. 29. Júnior OR, Borba AM, Alves CAF et al. Carnoy's solution over the inferior alveolar nerve as a complementary treatment for keratocystic odontogenic tumors. Rev Clin Pes Odontol 2007; 3(3): 199-202.

30. Al-Moraissi EA, Dahan AA, Alwadeai MS et al. What surgical treatment has the lowest recurrence rate following the management of keratocystic odontogenic tumor? A large systematic review and meta-analysis. J Craniomaxillofac Surg 2017; 45(1): 131-144. doi: 10.1016/J jcms.2016.10.013.

31. Chrcanovic BR, Gomez RS. Recurrence probability for keratocystic odontogenic tumors: an analysis o 6427 cases. J Craniomaxillofac Surg 2017; 45(2): 244-251. doi: 10.1016/j.jcms.2016.11.010.

32. Lebedev W, Butsan SB. The use of Carnoy's solution and its modifications for reducing the number of re currences after surgical removal of keratocystic odontogenic tumors and ameloblastomas: a systematic re view. Moscow Uni Biol Sci Bull 2019; 74(2): 108-116. doi: 10.3103/S0096392519020068

33. Alchalabi NJ, Merza AM, Issa SA. Using Carnoy's solution in treatment of keratocystic odontogenic tumor. Ann Maxillofac Surg 2017; 7(1): 51-56. doi: 10.4103/ams. ams_127_16.

34. Blanas N, Freund B, Schwartz M et al. Systematic review of the treatment and prognosis of the odontogenic keratocyst. Oral Surg Oral Med Oral Pathol Ora Radiol Endod 2000; 90(5): 553-558. doi: 10.1067/moe. 2000.110814.

35. Warburton G, Shihabi A, Ord RA. Keratocystic odontogenic tumor (KCOT/OKC) - clinical guidelines for re section. J Maxillofac Oral Surg 2015; 14(3): 558-564. doi: 10.1007/s12663-014-0732-7.

36. Titinchi F, Nortje CJ. Keratocystic odontogenic tumor: a recurrence analysis of clinical and radiographic parameters. Oral Surg Oral Med Oral Pathol Oral Radiol 2012 114(1): 136-142. doi: 10.1016/j.00oo.2012.01.032.

37. Agrawal R, Sangle A, Vyawahare A. Bismuth iodoform and paraffin paste: a boon in treatment of keratocystic odontogenic tumor: a case report. Int J Dent Med Res 2014; 1(2): 32-35

38. Borgonovo AE, Di Lascia S, Grossi G et al. Two-stage treatment protocol of keratocystic odontogenic tumour in young patients with Gorlin-Goltz syndrome: marsupialization and later enucleation with peripheral ostectomy. A 5-year-follow-up experience. Int J Pediatr Otorhinolaryngol 2011; 75(12): 1565-1571. doi: 10.1016/j. ijporl.2011.09.009

39. Cassoni A, Valentini V, Della Monaca M et al. Keratocystic odontogenic tumor surgical management: retrospective analysis on 77 patients. Eur J Inflamm 2014; 12(1): 209-215. doi: 10.1177/1721727X1401200123.

40. Davoodi P, Soufi L, Rezaei-soufi L et al. Submandibular abscess due to an infected keratocystic odontogenic tumor associated with simultaneous occurrence of a traumatic bone cyst: a rare case report. J Contemp Dent Pract 2013; 14(1): 133-136. doi: 10.5005/jp-journals-10024-1286.

41. Deboni MCZ, Brozoski MA, Traina AA et al. Surgical management of dentigerous cyst and keratocystic odontogenic tumor in children: a conservative approach and 7-year follow-up. J App Oral Sci 2012; 20(2): 282-285. doi: 10.1590/s1678-77572012000200025.

42. de Molon RS, Verzola MH, Pires LC et al. Five years follow-up of a keratocyst odontogenic tumor treated by marsupialization and enucleation: a case report and literature review. Contemp Clin Dent 2015; 6(Suppl 1): S106S110. doi: 10.4103/0976-237X.152963.

43. Ebenezer $V$, Ramalingam B. Importance of different modalities of treatment for the management of keratocystic odontogenic tumour with five year follow-up. J Clin Diagn Res 2014; 8(3): 225-228. doi: 10.7860/JCDR/2014/7486.4168.

44. Güler N, Sençift K, Demirkol Ö. Conservative management of keratocystic odontogenic tumors of jaws. ScientificWorldJournal 2012; 2012: 680397. doi: 10.1100/2012/680397.

45. Gao L, Wang XL, Li SM et al. Decompression as a treatment for odontogenic cystic lesions of the jaw. J Oral Maxillofac Surg 2014; 72(2): 327-333. doi: 10.1016/j. joms.2013.07.035

46. Caixeta Guimarães A, Dutra de Cassia Ferreira Santos M, Machado de Carvalho G et al. Giant keratocystic odontogenic tumor: three cases and literature review. Iran J Otorhinolaryngol 2013; 25(73): 245-252

47. Hasheminia D, Naemi V, Naghdi N. Conservative treatment protocol of keratocystic odontogenic tumor: report of a case with nevoid Basal cell carcinoma syndrome and literature review. J Maxillofac Oral Surg 2015; 14(Suppl 1): 475-481. doi: 10.1007/s12663-014-0689-6.

48. Kebede B, Dejene D, Teka A et al. Big keratocytic odontogenic tumor of the mandible: a case report. Ethiop J Health Sci 2016; 26(5): 491-496. doi: 10.4314/ejhs. v26i5.12.

49. Kim SH, Oh MS, Seo YS et al. Conservative treatment of multiple keratocystic odontogenic tumors in a young patient with nevoid Basal cell carcinoma syndrome by decompression: a 7-year follow-up study. J Clin Pediatr Dent 2017: 41(4): 300-304. doi: 10.17796/1053-4628-41.4.300. 50. Koçak-Berberoğlu H, Çakarer S, Brkić A et al. Three-dimensional cone-beam computed tomography for diagnosis of keratocystic odontogenic tumours; evaluation of four cases. Med ral Patol Oral Cir Bucal 2012; 17(6): e1000e1005 doi: 10.4317/medoral.17629.

51. Kunihiro T, Kawana H, Kodaka R et al. Keratocystic odontogenic tumor invading the maxillary sinus: a case report of collaborative surgery between an oral surgeon and an otorhinolaryngologist. J UOEH 2014; 36(4): 251256. doi: 10.7888/juoeh.36.251

52. Lacarbonara M, Marzo G, Lacarbonara V et al. Presentation of a keratocystic odontogenic tumor with agenesis: a case report. J Med Case Rep 2014; 8: 126. doi: 10.1186/1752-1947-8-126. 
53. Ledderhof NJ, Caminiti MF, Bradley G et al. Topical 5 -fluorouracil is a novel targeted therapy for the keratocystic odontogenic tumor. J Oral Maxillofac Surg 2017; 75(3): 514-524. doi: 10.1016/j.joms.2016.09.039.

54. Liu B, Cai Y, Wang SP et al. Recurrent keratocystic odontogenic tumor in the masseter muscle overlying the boney perforations: a case report. Oral Surg Oral Med Oral Pathol Oral Radiol 2012; 113(4): e1-5. doi: 10.1016/j.tripleo.2011.07.005

55. Zhao Y, Liu B, Cheng G et al. Recurrent keratocystic odontogenic tumours: report of 19 cases. Dentomaxillofac Radiol 2012; 41(2): 96-102. doi: 10.1259/dmfr/22891 281.

56. Morais de Melo W, Pereira-Santos D, Sonoda CK et al. Decompression for management of keratocystic odontogenic tumor in the mandible. J Craniofac Surg 2012; 23(6): e639-e640. doi: 10.1097/SCS.0b013e31827101e9.

57. Motwani MB, Mishra SS, Anand RM et al. Keratocystic odontogenic tumor: case reports and review of literature. J Indian Academy Oral Med Radiol 2011; 23(2): 150-154. doi: 10.5005/jp-journals-10011-1117.

58. Naruse T, Yamashita K, Yanamoto S et al. Histopathological and immunohistochemical study in keratocystic odontogenic tumors: predictive factors of recurrence. Oncol Lett 2017; 13(5): 3487-3493. doi: 10.3892/ol.2017. 5905.

59. Nomura K, Arakawa K, Fujishima F et al. Minimally invasive treatment for hard palate-invading maxillary keratocystic odontogenic tumor. Tohoku J Exp Med 2015; 237(4): 267-272. doi: 10.1620/tjem.237.267.

60. Ohki M. Transnasal marsupialization using endoscopic sinus surgery for treatment of keratocystic odontogenic tumor in maxillary sinus. Case Rep Otolaryngol 2012 2012: 281402. doi: 10.1155/2012/281402.

61. Ribeiro Junior O, Borba AM, Alves CA et al. Keratocystic odontogenic tumors and Carnoy's solution: results and complications assessment. Oral Dis 2012; 18(6): 548-557. doi: 10.1111/j.1601-0825.2012.01907.x.

62. Roopak B, Singh M, Shah A et al. Keratocystic odontogenic tumor: treatment modalities: study of 3 cases. Niger J Clin Pract 2014; 17(3): 378-383. doi: 10.4103/11193077.130251.

63. Scartezini GR, Oliveira GC, Guedes OA et al. Diagnostic and treatment features of keratocystic odontogenic tumors. Stomatos 2012; 18(35): 9-15.

64. Schussel JL, Stramandinoli RT, Dissenha JL et al. Retrospective study of 25 cases of keratocystic odontogenic tumor: epidemiology and treatment. J Contemp Dent Pract 2011; 12(2): 100-103. doi: 10.5005/jp-jour nals-10024-1016

65. Bharani KS, Lakshmi SS, Kamath AR et al. Keratocystic odontogenic tumor: case report, treatment review and future diagnostic trends. J Oral Maxillofacial Surg Med Pathol 2013; 25(1): 85-92.

66. Srivatsan K, KumarV, Mahendra A et al. Bilateral keratocystic odontogenic tumor: a report of two cases. Nat J Maxillofac Surg 2014; 5(1): 86-89. doi: 10.4103/09755950.140186.

67. Sivanmalai S, Kandhasamy K, Prabu N et al. Carnoy's solution in the mangement of odontogenic keratocyst. J Pharm Bioallied Sci 2012; 4 (Suppl 2): S183-S185. doi: 10.4103/0975-7406.100266

68. Vázquez-Romero MD, Serrera-Figallo ML, Alberdi-Navarro J et al. Maxillary peripheral keratocystic odontogenic tumor. A clinical case report. J Clin Exp Dent 2017; 9(1): e167-e171. doi: 10.4317/jced.53438.

69. Warburton G, Shihabi A, Ord RA. Keratocystic odontogenic tumor (KCOT/OKC) -clinical guidelines for resection. J Maxillofac Oral Surg 2015; 14(3): 558-564. doi: 10.1007/s12663-014-0732-7.

70. Zhou J, Wang L, Chen Z et al. Giant keratocystic odontogenic tumor of the maxillary sinus and zygoma: a case report. Oncol Lett 2014; 8(6): 2675-2677. doi: 10.3892/ol.2014.2576.

71. Yang S-I, Park Y-I, Choi S-Y et al. A retrospective study of 220 cases of keratocystic odontogenic tumor (KCOT) in 181 patients. Asian J Oral Maxillofac Surg 2011; 23(3): 117121. doi: 10.1016/j.ajoms.2011.03.002.

72. Yildirim G, Ataoglu H, Kalayci A et al. Conservative treatment protocol for keratocystic odontogenic tumour: a follow-up study of 3 cases. J Oral Maxillofac Res 2010; 1(3): e7. doi: 10.5037/jomr.2010.1307.

73. Jafaripozve N, Jafaripozve S, Khorasgani MA. Kerathocyst odontogenic tumor: importance of selection the best treatment modality and a periodical follow-up to prevent from recurrence: a case report and literature review. Int J Prev Med 2013; 4(8): 967-970.

74. Ribeiro-Júnior O, Borba AM, Alves CAF et al. Reclassification and treatment of odontogenic keratocysts: a cohort study. Braz Oral Res 2017; 31: e98. doi: 10.1590/18073107bor-2017.vol31.0098.

75. Oliveira A, Ferrisse T, Pereira-Filho V et al. Treatment of a large keratocystic odontogenic tumor in a mandible using decompression technique followed by enucleation. Oral Surg Oral Med Oral Pathol Oral Radiol Endodontol 2017; 124(2): e96. doi: 10.1016/j.000o.2017.05.187. 